\title{
Primal Explicit Max Margin Feature Selection for Nonlinear Support Vector Machines
}

\author{
Aditya Tayal ${ }^{\mathrm{a}, 1, *}$, Thomas F. Coleman ${ }^{\mathrm{b}, 1,2}$, Yuying $\mathrm{Li}^{\mathrm{a}, 1}$ \\ ${ }^{a}$ Cheriton School of Computer Science, University of Waterloo, Waterloo, ON, Canada N2L 3G1 \\ ${ }^{b}$ Combinatorics and Optimization, University of Waterloo, Waterloo, ON, Canada N2L $3 G 1$
}

\begin{abstract}
Embedding feature selection in nonlinear SVMs leads to a challenging non-convex minimization problem, which can be prone to suboptimal solutions. This paper develops an effective algorithm to directly solve the embedded feature selection primal problem. We use a trust-region method, which is better suited for non-convex optimization compared to line-search methods, and guarantees convergence to a minimizer. We devise an alternating optimization approach to tackle the problem efficiently, breaking it down into a convex subproblem, corresponding to standard SVM optimization, and a non-convex subproblem for feature selection. Importantly, we show that a straightforward alternating optimization approach can be susceptible to saddle point solutions. We propose a novel technique, which shares an explicit margin variable to overcome saddle point convergence and improve solution quality. Experiment results show our method outperforms the state-of-the-art embedded SVM feature selection method, as well as other leading filter and wrapper approaches.
\end{abstract}

Keywords: nonlinear feature selection, support vector machine, non-convex optimization, trust-region method, alternating optimization

\footnotetext{
${ }^{*}$ Corresponding author

Email addresses: amtayal@uwaterloo.ca (Aditya Tayal), tf coleman@uwaterloo.ca (Thomas F. Coleman), yuying@uwaterloo.ca (Yuying Li)

${ }^{1}$ All three authors acknowledge funding from the National Sciences and Engineering Research Council of Canada

${ }^{2}$ This author acknowledges funding from the Ophelia Lazaridis University Research Chair. The views expressed herein are solely from the authors.
} 


\section{Introduction}

Feature selection has become a significant research focus in statistical machine learning and data mining communities. As increasingly more data is available, problems with hundreds and thousands of features have become common. Some examples include text processing of internet documents, gene micro-array analysis, combinatorial chemistry, economic forecasting and context based collaborative filtering. However, irrelevant and redundant features reduce the effectiveness of data mining and may detract from the quality and accuracy of the resulting model. The goal of feature selection is to identify the most relevant subset of input features for the learning task, improving generalization error and model interpretability.

In this paper, we focus on feature selection for nonlinear Support Vector Machine (SVM) classification. SVM is based on the principle of maximum-margin separation, which achieves the goal of Structural Risk Minimization by minimizing a generalization bound on model complexity and training error concurrently (Cortes and Vapnik, 1995; Vapnik, 1998). The model is obtained by solving a convex quadratic programming problem. Linear SVM models can be extended to nonlinear ones by transforming the input features using a set of nonlinear basis functions. An important advantage of the SVM is that the transformation can be done implicitly using the "kernel trick", thereby allowing even infinite-dimensional feature expansions (Boser et al., 1992). Empirically, SVMs have performed extremely well in diverse domains (e.g. see Byun and Lee, 2002; Schölkopf et al., 2004).

Determining the optimal set of input features is in general NP-hard, requiring an exhaustive search of all possible subsets. Practical alternatives can be grouped into filter, wrapper, and embedded techniques (Guyon and Elisseeff, 2003). Filter methods operate independently of the SVM classifier to score features according to how useful they are in predicting the output. Relief (Kira and Rendell, 1992; Šikonja and Kononenko, 2003) is a popular multivariate nonlinear filter that has successfully been used as a preprocessing step for SVMs (e.g. see Marchiori, 2005). Wrapper methods, on the other hand, use the SVM classifier to guide the search in the space of all possible subsets. For instance the most common wrapper, recursive feature elimination, greed- 
ily removes the worst (or adds the best) feature according to the loss (or gain) of the SVM classifier at each iteration (Guyon et al., 2002). Finally, embedded approaches incorporate the feature selection criterion in the SVM objective itself. Embedded methods can offer significant advantages over filters and wrappers, since they tightly couple feature selection with SVM learning, simultaneously searching over the feature and model space.

For linear SVMs, several embedded feature selection methods have been proposed. The general idea is to incorporate sparse regularization of the primal weight vector (for example see Bradley and Mangasarian, 1998; Zhu et al., 2003; Weston et al., 2003; Fung and Mangasarian, 2004; Chan et al., 2007; Tan et al., 2010). However, similar techniques cannot be readily applied to nonlinear SVM classifiers, since the weight vector is not explicitly formed. Sparse regularization of the dual variables (support vectors) lead to a reduction in the number of kernel functions needed to generate the nonlinear surface, but does not result in a reduction of input features (Fung and Mangasarian, 2004).

Embedding feature selection in a nonlinear SVM requires optimizing over additional parameters in the kernel function. This can be viewed as an instance of Generalized Multiple Kernel Learning (GMKL) (Varma and Babu, 2009), which offers the state-of-the-art solution for embedded nonlinear feature selection. However, the resulting problem is non-convex. The algorithm proposed in Varma and Babu (2009) to solve GMKL is based on gradient descent, i.e. line-search along the negative gradient. Hence, it uses a first-order convex approximation at each iterate, which can fail to find a minimizer when the problem is non-convex. In contrast, trust-region algorithms are better suited for non-convex optimization. At each iterate they solve non-convex second-order approximations with guaranteed convergence to a minimizer.

This paper develops an effective algorithm to solve the non-convex optimization problem that results from embedding feature selection in nonlinear SVMs. Our contributions in this paper are as follows:

1. We invoke Representor Theorem to formulate a primal embedded feature selection SVM problem and use a smoothed hinge loss function to obtain a simpler 
bound constrained problem. We solve the resulting problem using a generalized trust-region algorithm for bound constrained minimization.

2. To improve efficiency we propose a two-block alternating optimization scheme, in which we iteratively solve (a) standard SVM problem and (b) a smaller nonconvex feature selection problem. Importantly, we propose a novel technique that uses an explicit margin variable, which is shared between subproblems. This helps avoid suboptimal local minima. Moreover, by focussing on maximizing margin in the feature selection problem - a critical quantity for generalization error-we are able to further improve solution quality.

3. We compare our methods to GMKL and other leading nonlinear feature selectors, and show that our approach improves results.

The rest of the paper is organized as follows. Section 2 formulates the embedded feature selection problem. Section 3 describes the bound constrained trust-region approach to solve the problem in the full feature and model space. Section 4 develops the explicit margin alternating optimization approach. Section 5 compares our approach with other nonlinear feature selection methods on several datasets and we conclude with a discussion in Section 6.

\section{Feature selection in nonlinear SVMs}

We start by describing the embedded feature selection problem for nonlinear SVMs. We motivate and explain the formulation with respect to margin-based generalization bounds.

Consider a set of $n$ training points, $\mathbf{x}_{i} \in \mathbb{R}^{d}$, and corresponding class labels, $y_{i} \in$ $\{+1,-1\}, i=1, \ldots, n$. Each component of $\mathbf{x}_{i}$ is an input feature. In classical SVM, proposed by Cortes and Vapnik (1995), a linear classifier $(\mathbf{w}, b)$ is learned by maximizing the geometric margin, defined as $\gamma \equiv \min _{i} y_{i}\left(\mathbf{w}^{T} \mathbf{x}_{i}+b\right) /\|\mathbf{w}\|$, where $\|\cdot\|$ denotes 2-norm. Since the decision hyperplane associated with $(\mathbf{w}, b)$ does not change upon rescaling to $(\lambda \mathbf{w}, \lambda b)$, for $\lambda \in \mathbb{R}^{+}$, the function output at the margin (functional margin) is fixed to 1 ; geometric margin is given by $\gamma=1 /\|\mathbf{w}\|$, and the norm of the weight 
vector is minimized. Thus in the standard setting, SVM results in the following convex quadratic programming problem:

$$
\begin{array}{ll}
\min _{\mathbf{w}, b, \boldsymbol{\xi}} & \frac{1}{2}\|\mathbf{w}\|^{2}+C \sum_{i=1}^{n} \xi_{i}, \\
\text { s.t. } & y_{i}\left(\mathbf{w}^{T} \mathbf{x}_{i}+b\right) \geq 1-\xi_{i}, \quad i=1, \ldots, n, \\
& \xi_{i} \geq 0, \quad i=1, \ldots, n .
\end{array}
$$

Here, $\xi_{i}$ 's are margin violations, and $C$ is a penalty controlling the trade-off between empirical error and (implicitly computed) geometric margin.

To obtain a non-linear decision function, the kernel trick (Boser et al., 1992) is used by defining a kernel function, $K\left(\mathbf{x}, \mathbf{x}^{\prime}\right) \equiv \phi(\mathbf{x})^{T} \phi\left(\mathbf{x}^{\prime}\right)$, where $K: \mathbb{R}^{d} \times \mathbb{R}^{d} \rightarrow \mathbb{R}$ and $\phi: \mathbb{R}^{d} \rightarrow F$ is a non-linear map from input features to a (potentially infinite dimensional) derived feature space. A kernel function, satisfying Mercer's condition (Mercer, 1909; Courant and Hilbert, 1953), directly computes the inner product of two vectors in a derived feature space, without the need to explicitly determine the feature mapping. Conventionally, the kernel is used in the dual of problem (1), where all occurrences of data appear inside an inner product. However, we can also formulate the primal problem in the derived feature space by expressing the weight vector as a linear combination of mapped data points, $\mathbf{w}=\sum_{i=1}^{n} y_{i} u_{i} \phi\left(\mathbf{x}_{i}\right)$, due to Representer theorem (Scholkopf and Smola, 2002). We denote the coefficients as $u_{i}$, and not $\alpha_{i}$ as used in the standard SVM literature, in order to distinguish them from the typical Lagrange multiplier interpretation. Substituting this form in (1) leads to the following primal non-linear SVM problem,

$$
\begin{aligned}
\min _{\mathbf{u}, b, \boldsymbol{\xi}} & \frac{1}{2} \sum_{i, j=1}^{n} y_{i} y_{j} u_{i} u_{j} K\left(\mathbf{x}_{i}, \mathbf{x}_{j}\right)+C \sum_{i=1}^{n} \xi_{i}, \\
\text { s.t. } & y_{i}\left(\sum_{j=1}^{n} y_{j} u_{j} K\left(\mathbf{x}_{i}, \mathbf{x}_{j}\right)+b\right) \geq 1-\xi_{i}, i=1, \ldots, n, \\
& \xi_{i} \geq 0, i=1, \ldots, n .
\end{aligned}
$$

The geometric margin in the derived feature space is given by

$$
\gamma=\frac{1}{\sqrt{\sum_{i, j=1}^{n} y_{i} y_{j} u_{i} u_{j} K\left(\mathbf{x}_{i}, \mathbf{x}_{j}\right)}} .
$$


The dual of problem (2) reveals that the primal variable $u_{i}$ is equivalent to the standard SVM dual Lagrange multiplier $\alpha_{i}$, i.e. $u_{i}=\alpha_{i}$, when the kernel matrix is non-singular. If the kernel matrix is singular, then the coefficient expansion $u_{i}$ is not unique (even though the decision function is) and solving (2) will produce one of the possible expansions, of which $\alpha_{i}$ is also a minimizer.

The maximum margin classifier is motivated by theoretical bounds on the generalization error. Specifically, Vapnik (1998) shows that generalization error for $n$ points is bounded by,

$$
e r r \leq \frac{c}{n}\left[\left(\frac{R^{2}}{\gamma^{2}}+\|\boldsymbol{\xi}\|^{2}\right) \log ^{2} n+\log \frac{1}{\delta}\right]
$$

for some constant $c$ with probability $1-\delta$, where $\gamma$ is the geometric margin of the classifier. The key expression, on which generalization depends, is $R^{2} / \gamma^{2}+\|\boldsymbol{\xi}\|^{2}$, where $\xi$ is the margin slack vector (normalized by $\gamma$ ), and $R$ is the radius of the ball that encloses the set of points in the derived feature space, $\left\{\phi\left(\mathbf{x}_{i}\right)\right\}_{i=1}^{n}$. For a fixed dataset and kernel choice, $R$ is constant, and thus maximizing the margin while reducing margin violations minimizes the upper bound in (3). Although the generalization bound suggests using a 2-norm penalty on margin violations, a 1-norm penalty is preferred for classification tasks, since it is a better approximation to a step penalty (Vapnik, 1998).

Now consider learning such a classifier while allowing input features to be weighted according to their relevance. We introduce a feature weight vector, $\mathbf{z} \in \mathbb{R}^{d}$, where $z_{l} \geq 0$ is a weight applied to input feature $l .^{3}$ For convenience we define a diagonal matrix, $Z \in \mathbb{R}^{d \times d}$ with $Z_{l l}=z_{l}$. Hence, weighted points are mapped to $\phi(Z \mathbf{x})$ and we can replace $K\left(\mathbf{x}, \mathbf{x}^{\prime}\right)$ by $K\left(Z \mathbf{x}, Z \mathbf{x}^{\prime}\right)$ in problem (2) to obtain the following embedded feature selection problem, in which we simultaneously search for optimal feature weights, $\mathbf{z}$,

\footnotetext{
${ }^{3}$ Without loss of generality, we assume features have been normalized to unit variance
} 
while solving for model parameters, $(\mathbf{u}, b)$ :

$$
\begin{aligned}
\min _{\mathbf{u}, b, \boldsymbol{\xi}, \mathbf{z}} & \frac{1}{2} \sum_{i, j=1}^{n} y_{i} y_{j} u_{i} u_{j} K\left(Z \mathbf{x}_{i}, Z \mathbf{x}_{j}\right)+C \sum_{i=1}^{n} \xi_{i}+\mu\|\mathbf{z}\|_{1}, \\
\text { s.t. } & y_{i}\left(\sum_{j=1}^{n} y_{j} u_{j} K\left(Z \mathbf{x}_{i}, Z \mathbf{x}_{j}\right)+b\right) \geq 1-\xi_{i}, i=1, \ldots, n, \\
& \xi_{i} \geq 0, i=1, \ldots, n, \\
& z_{l} \geq 0, l=1, \ldots, d .
\end{aligned}
$$

We include 1-norm regularization of feature weights, $\mathbf{z}$, with a penalty parameter $\mu>0$. This serves two purposes. Firstly, the 1-norm regularizer has the beneficial effect of suppressing variables to produce a sparse set of non-zero feature weights (Tibshirani, 1996). This property is desirable for feature selection where we are interested in identifying the most useful subset of input features. Secondly, it acts to minimize the radius of the enclosing ball, $R$, for the generalization bound in (3). Given two feature weight vectors, $\mathbf{z}$ and $\mathbf{z}^{\prime}$, if $z_{l}^{\prime} \leq z_{l}$, for $l=1, \ldots, d$, then $\sum_{l} z_{l}^{\prime 2}\left(x_{i l}-x_{j l}\right)^{2} \leq \sum_{l} z_{l}^{2}\left(x_{i k}-x_{j l}\right)^{2}$, implying $\left\|Z^{\prime} \mathbf{x}_{i}-Z^{\prime} \mathbf{x}_{j}\right\| \leq\left\|Z \mathbf{x}_{i}-Z \mathbf{x}_{j}\right\|$. Thus suppressing feature weights reduces distances between points in input space, which in turn results in a smaller enclosing ball

in feature space. To minimize the generalization bound, we solve (4) and calibrate margin, errors, and radius via parameters $C$ and $\mu$, which can be determined by crossvalidation.

\subsection{Relation to GMKL}

We note that problem (4) can be viewed as an instance of generalized multiple kernel learning (Varma and Babu, 2009). For example, if we consider a radial basis kernel, then weighting features is equivalent to considering a product of 1-dimensional radial basis kernels derived from individual features with different width parameters. To solve this optimization problem Varma and Babu (2009) propose a method based on gradient descent. The algorithm follows Chapelle et al. (2002) by reformulating the problem as a nested two step optimization: in an outer loop, the width parameters (i.e. feature weights) are updated by a line search step along the negative gradient assuming fixed SVM model parameters, while in an inner loop, the kernel is held fixed and SVM 
model parameters are updated. Assuming a 1-norm feature weight regularizer, in the outer loop GMKL solves

$$
\min _{\mathbf{z}} F(\mathbf{z})+\mu\|\mathbf{z}\|_{1} \text { subject to } z_{l} \geq 0, l=1, \ldots, d
$$

where

$$
\begin{aligned}
F(\mathbf{z})=\max _{\alpha} & \sum_{i=1}^{n} \alpha_{i}-\frac{1}{2} \sum_{i, j=1}^{n} \alpha_{i} \alpha_{j} y_{i} y_{j} K\left(Z \mathbf{x}_{i}, Z \mathbf{x}_{j}\right) \\
\text { s.t. } & \sum_{i==1}^{n} y_{i} \alpha_{i}=0, \\
& 0 \leq \alpha_{i} \leq C, i=1, \ldots, n,
\end{aligned}
$$

is the solution of the dual SVM problem for fixed feature weights, which is solved in an inner loop. If $\alpha^{*}$ solves (6) exactly, the gradient, $\nabla_{z} F$, can be determined as a function of optimal $\alpha^{*}$ due to Danskin's Theorem (Danskin, 1967). At each iteration, a projected Armijo-step (i.e. line search) is taken in the direction of negative gradient to minimize (5). $F(\mathbf{z})$ is a non-convex function. The gradient descent algorithm uses a local first-order convex approximation and does not guarantee convergence to a minimizer in the non-convex case. Moreover, only an approximation to a gradient is available since the gradient requires an exact solution to the SVM problem, which computationally cannot be achieved. In contrast, we use a trust region based algorithm to solve the problem, which is better suited for non-convex optimization (4) and guarantees convergence to a minimizer.

\section{Solving the full-space feature selection problem}

In this section, we solve the embedded feature selection SVM problem using trust region algorithm for a bound constrained problem. Problem (4) can be written as:

$$
\min _{\mathbf{u}, b, \mathbf{z}} \Omega(\mathbf{u}, b, \mathbf{z}) \text { s.t. } z_{l} \geq 0, \quad l=1, \ldots, d,
$$

where the objective is expressed in exact-penalty form:

$$
\Omega(\cdot)=\frac{1}{2} \sum_{i, j=1}^{n} y_{i} y_{j} u_{i} u_{j} K\left(Z \mathbf{x}_{i}, Z \mathbf{x}_{j}\right)+C \sum_{i=1}^{n} V\left(y_{i}, f\left(\mathbf{x}_{i}\right)\right)+\mu\|\mathbf{z}\|_{1}
$$




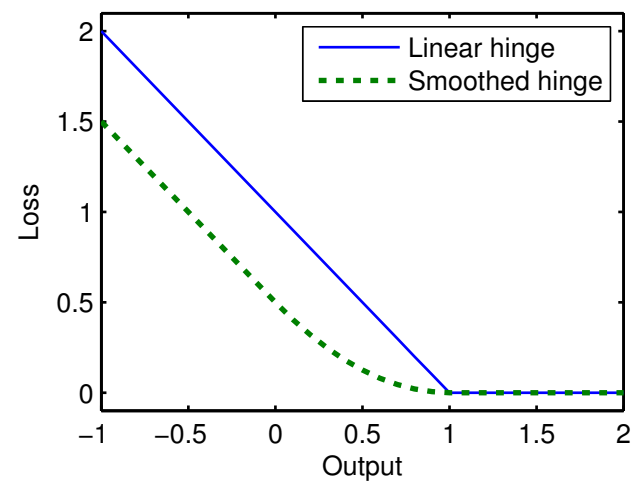

Figure 1: The smoothed hinge loss is a differentiable approximation of the linear hinge loss aligned at the margin. Here a smoothed hinge loss is shown with $\varepsilon=0.5$.

Here, $f\left(\mathbf{x}_{i}\right)=\sum_{j=1}^{n} y_{j} u_{j} K\left(Z \mathbf{x}_{i}, Z \mathbf{x}_{j}\right)+b$ is the decision function, and $V\left(y_{i}, f\left(\mathbf{x}_{i}\right)\right)=$ $\max \left(0,1-y_{i} f\left(\mathbf{x}_{i}\right)\right)$ is a non-differentiable linear hinge loss function. Alternative differentiable loss functions can be used instead. In this paper we use the following $\varepsilon$ smoothed hinge loss function $V_{\mathcal{\varepsilon}}\left(y_{i}, f\left(\mathbf{x}_{i}\right)\right)$ :

$$
V_{\varepsilon}\left(y_{i}, f\left(\mathbf{x}_{i}\right)\right) \equiv \begin{cases}\left(1-y_{i} f\left(\mathbf{x}_{i}\right)\right)-\varepsilon & \text { if } y_{i} f\left(\mathbf{x}_{i}\right)<1-2 \varepsilon \\ \frac{1}{4 \varepsilon}\left(1-y_{i} f\left(\mathbf{x}_{i}\right)\right)^{2} & \text { if } 1-2 \varepsilon \leq y_{i} f\left(\mathbf{x}_{i}\right)<1 \\ 0 & \text { if } y_{i} f\left(\mathbf{x}_{i}\right) \geq 1 .\end{cases}
$$

The loss function transitions from linear cost to zero cost using a quadratic segment and bears similarity to a (truncated) Huber loss (see Figure 1). Thus problem (7) becomes a smooth minimization problem with simple bound constraints. In our experiments we set $\varepsilon=0.5$. From a classification perspective, the smoothed hinge loss function is asymptotically margin-maximizing (Rosset et al., 2003) and Bayes-risk consistent (Nguyen et al., 2009), and offers similar benefits as a linear hinge loss.

\subsection{Trust region algorithm}

Trust region algorithms are a class of relatively new optimization algorithms compared to classical line search methods. The main difference can be explained as follows. In the trust region method, we choose a step size (the size of the trust region) first and then search for a step direction, while in line search methods, we first choose 
a descent direction and then a step size. The trust region is usually a spherical or elliptical neighborhood centered at the current iterate, in which a local second order Taylor expansion (i.e. quadratic approximation) of the objective can be trusted. One of the main advantages of trust region methods is that a global solution to the local quadratic model can be computed, even when the Hessian is indefinite (non-convex). As a result trust region algorithms are better suited for non-convex optimization and can guarantee convergence to a minimizer.

For unconstrained minimization, the trust region method solves the following subproblem to obtain the step-size, $\mathbf{s}$, given the current iterate $\mathbf{x}^{(p)}$ :

$$
\begin{aligned}
\min _{\mathbf{s} \in \mathbb{R}^{m}} & \mathbf{s}^{T} \mathbf{g}^{(p)}+\frac{1}{2} \mathbf{s}^{T} H^{(p)} \mathbf{s}, \\
\text { s.t. } & \|\mathbf{s}\|_{2} \leq \Delta^{(p)} .
\end{aligned}
$$

Here $\mathbf{g}^{(p)}$ and $H^{(p)}$ are the gradient and Hessian of the objective function at $\mathbf{x}^{(p)}$, and $\Delta^{(p)}$ is the current radius of the trust region. For a nonconvex minimization problem, the Hessian $H^{(p)}$ can be indefinite and (9) is a nonconvex quadratic minimization problem with a ball constraint. A global minimizer of this subproblem can be computed since there is no duality gap for a trust region subproblem. For example, assuming $\Delta^{(p)}=1$, the dual of (9) can be solved by first computing a solution to a convex 1-dimensional problem:

$$
\begin{aligned}
\max _{v \in \mathbb{R}} & -\sum_{i=1}^{m} \frac{\left(\mathbf{q}_{i}^{T} \mathbf{g}^{(p)}\right)^{2}}{v_{i}+v}-v, \\
\text { s.t. } & v \geq-v_{\min }\left(H^{(p)}\right),
\end{aligned}
$$

where $v_{i}$ and $\mathbf{q}_{i}$ are the eigenvalues and corresponding orthonormal eigenvectors of $H^{(p)}$, respectively, and $v\left(H^{(p)}\right)$ denotes the minimum eigenvalue of $H^{(p)}$ (Boyd and Vandenberghe, 2004).

For our implementation, we use the trust region method described in Coleman and Li (1996), which generalizes the unconstrained case to bound constraints. Each iteration requires an eigen-decomposition of the Hessian matrix involving cubic complexity. Consequently, solving (7) requires $O\left((n+d)^{3}\right)$ operations at each iteration. In the next section, we propose an explicit margin alternating optimization approach, which 
improves computation efficiency by breaking the problem down into two smaller subproblems, with $O\left(n^{3}\right)$ complexity for the SVM subproblem and $O\left(d^{3}\right)$ for the feature selection subproblem, while able to further improve solution quality by avoiding suboptimal local minima.

\section{A novel alternating optimization approach with explicit margin sharing}

We develop a novel alternating optimization (AO) method with explicit margin. We devise the formulation in three successive stages. First, we present a simple, but naive approach, which alternates between solving for SVM model parameters and feature weights. Second, we extend the problem with an explicit margin variable which is shared between AO subproblems. Finally, we relax the margin term so it is not tied to geometric margin when solving the feature selection subproblem.

\subsection{Simple $A O$}

For fixed feature weights, (7) reduces to a convex problem that corresponds to regular SVM optimization. The standard SVM problem can be solved efficiently (e.g. see Platt, 1999; Fan et al., 2005). To avail of this, we consider a two-block AO approach (also known as nonlinear block coordinate descent or Gauss-Seidel method), which iterates between 1) fixing feature weights and solving SVM for model parameters (u, $b)$, and 2) fixing model parameters and solving a smaller non-convex problem for feature weights, z. The procedure is outlined in Algorithm 1.

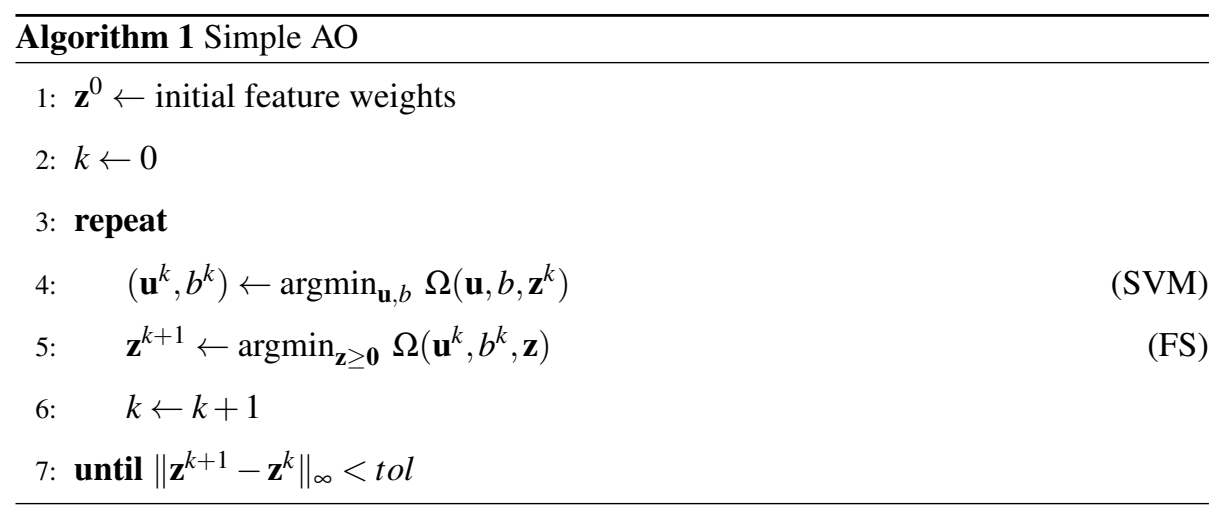


We can use any convex solver for the SVM subproblem and use the bound-constrained trust-region algorithm described in Section 3.1 to solve the non-convex feature selection subproblem. The procedure generates a sequence $\left\{\left(\mathbf{u}^{k}, b^{k}, \mathbf{z}^{k}\right)\right\}_{k=1}^{\infty}$, which can be shown to converge to a stationary point of (7) (Grippo and Sciandrone, 2000). We stop when successive changes in feature weights are less than a prespecified tolerance, tol.

Although this simple alternating optimization scheme improves computational efficiency by breaking the problem down into two smaller subproblems, it detracts from an important advantage of using the trust-region algorithm—convergence to a minimizer. Even though each subproblem converges to a minimizer when viewed along their restricted subspaces, the solution may not converge to a minimizer in the full variable space (Bezdek and Hathaway, 2002). A simple example can be illustrative. Consider minimizing the three-variable quadratic function,

$$
f\left(x_{1}, x_{2}, x_{3}\right)=\left(x_{1}+x_{2}-2\right)^{2}-3\left(x_{1}+x_{2}-2\right)\left(x_{3}-1\right)+\left(x_{3}-1\right)^{2},
$$

using $\mathrm{AO}$ on variable subsets $\left\{x_{1}, x_{2}\right\}$ and $\left\{x_{3}\right\}$. For fixed $x_{3}=1$, the point $\left(x_{1}, x_{2}\right)=$ $(1,1)$ is a global minimizer of $f\left(x_{1}, x_{2}, 1\right)=\left(x_{1}+x_{2}-2\right)^{2}$, and for the fixed point $\left(x_{1}, x_{2}\right)=(1,1), x_{3}=1$ is the global minimizer of $f\left(1,1, x_{3}\right)=\left(x_{3}-1\right)^{2}$. Consequently, $\mathrm{AO}$ can converge to $\left(x_{1}, x_{2}, x_{3}\right)=(1,1,1)$, which is a stationary point, but not a minimizer of the full variable space (i.e. it is a saddle point).

Indeed, in our experiments, we find the simple AO scheme is less effective for non-linear feature selection. We address this shortcoming by introducing an auxiliary margin variable, which is shared between the two subproblems.

\subsection{Margin sharing $A O$}

Returning to the simple three-variable example, we can introduce a perspective transformation, $x_{1}=\bar{x}_{1} / y$ and $x_{2}=\bar{x}_{2} / y$, to obtain,

$$
\bar{f}\left(\bar{x}_{1}, \bar{x}_{2}, x_{3}, y\right)=\left(\bar{x}_{1} / y+\bar{x}_{2} / y-2\right)^{2}-3\left(\bar{x}_{1} / y+\bar{x}_{2} / y-2\right)\left(x_{3}-1\right)+\left(x_{3}-1\right)^{2} .
$$

Instead of alternating between two disjoint sets of variables, we share the $y$ variable between AO iterates. Thus we minimize $\bar{f}\left(\bar{x}_{1}, \bar{x}_{2}, x_{3}, y\right)$ over variable subsets $\left\{\bar{x}_{1}, \bar{x}_{2}, y\right\}$ 
and $\left\{x_{3}, y\right\}$. For fixed $x_{3}=1$, we minimize $\bar{f}\left(\bar{x}_{1}, \bar{x}_{2}, 1, y\right)=\left(\bar{x}_{1} / y+\bar{x}_{2} / y-2\right)^{2}$, to obtain a global minimizer $\left(\bar{x}_{1}, \bar{x}_{2}, y\right)=(1,1,1)$ which corresponds to $\left(x_{1}, y_{1}\right)=(1,1)$ as before. However, for fixed $\left(\bar{x}_{1}, \bar{x}_{2}\right)=(1,1)$, we now minimize $\bar{f}\left(1,1, x_{3}, y\right)=4(1 / y-$ $1)^{2}-6(1 / y-1)\left(x_{3}-1\right)+\left(x_{3}-1\right)^{2}$ to find that the Hessian with respect to $\left(x_{3}, y\right)$ is indefinite at $\left(x_{3}, y\right)=(1,1)$. Thus by extending the subspace with an auxiliary perspective variable, which is shared between AO subproblems, we can avoid convergence to saddle points.

Motivated by this observation, we consider a perspective transformation of SVM model parameters in the AO approach. We substitute $\mathbf{u}=\overline{\mathbf{u}} / \lambda$ and $b=\bar{b} / \lambda$ in (4) to obtain,

$$
\begin{aligned}
\min _{\overline{\mathbf{u}}, \bar{b}, \boldsymbol{\xi}, \mathbf{z}, \lambda} & \frac{1}{2 \lambda^{2}} \sum_{i, j=1}^{n} y_{i} y_{j} \bar{u}_{i} \bar{u}_{j} K\left(Z \mathbf{x}_{i}, Z \mathbf{x}_{j}\right)+C \sum_{i=1}^{n} \xi_{i}+\mu\|\mathbf{z}\|_{1}, \\
\text { s.t. } & y_{i}\left(\sum_{j=1}^{n} y_{j} \bar{u}_{j} K\left(Z \mathbf{x}_{i}, Z \mathbf{x}_{j}\right)+\bar{b}\right) \geq \lambda-\lambda \xi_{i}, i=1, \ldots, n \\
& \xi_{i} \geq 0, \quad i=1, \ldots, n \\
& z_{l} \geq 0, \quad l=1, \ldots, d \\
\lambda & \geq 0
\end{aligned}
$$

Note, the auxiliary perspective variable, $\lambda$, is equivalent to the functional margin (see Section 2). We share $\lambda$ between the AO subproblems. For fixed fixture weights, (10) is equivalent to a regular SVM, as before (since we fix the functional margin, $\lambda$, to 1 to make the problem well-posed). However, in the feature selection subproblem, when model parameters $(\overline{\mathbf{u}}, \bar{b})$ are fixed, $\lambda$ provides an additional view of the margin component. This allows us to move along a direction in the SVM model space while solving the feature selection subproblem. As a result we can avoid convergence to a saddle point. The procedure is shown in Algorithm 2 using the following exact-penalty expression for the objective:

$$
\bar{\Omega}(\overline{\mathbf{u}}, \bar{b}, \mathbf{z}, \lambda)=\frac{1}{2 \lambda^{2}} \sum_{i, j=1}^{n} y_{i} y_{j} \bar{u}_{i} \bar{u}_{j} K\left(Z \mathbf{x}_{i}, Z \mathbf{x}_{j}\right)+C \sum_{i=1}^{n} V\left(y_{i}, \frac{f\left(\mathbf{x}_{i}\right)}{\lambda}\right)+\mu\|\mathbf{z}\|_{1} .
$$

We solve the extended feature selection (XFS) subproblem using the bound constrained trust region algorithm described in Section 3.1. We use $\mathbf{z}=\mathbf{z}^{k}$ and $\lambda=1$ as initial 
points in step 6. In our experiments we found that margin sharing AO yields similar performance to the full-space solution discussed in Section 3-with the added benefit of lower complexity.

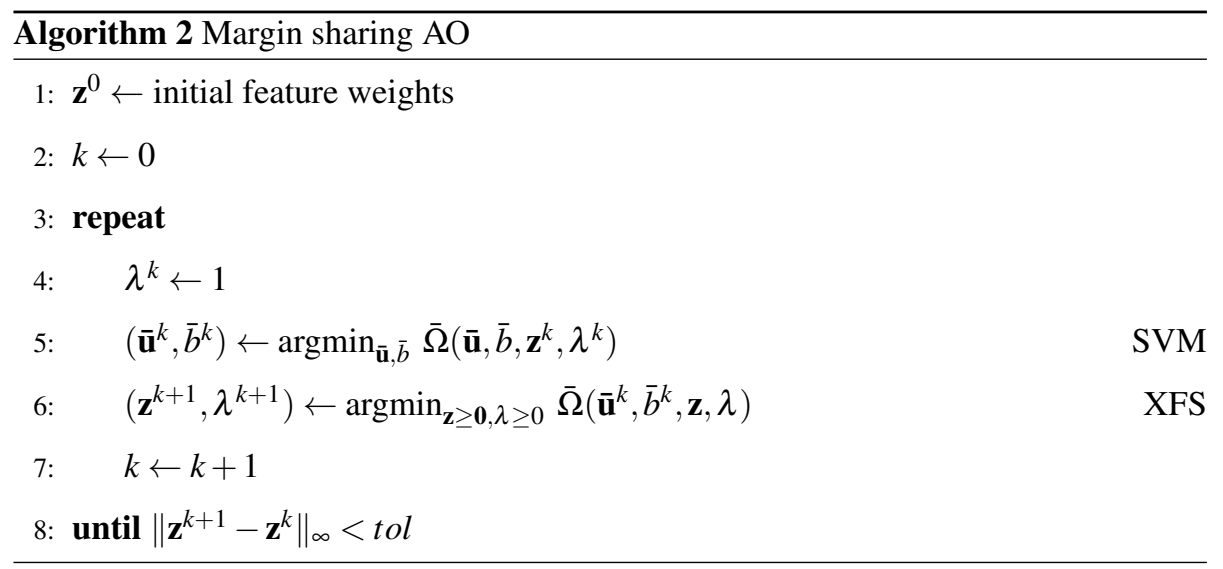

\subsection{Explicit (functional) margin $A O$}

We can further improve the solution by observing that for fixed support vectors, it is more relevant to maximize functional margin than geometric margin. Specifically, we propose to minimize the following objective (over $\mathbf{z}, \lambda$ ) in place of the subproblem (XFS).

$$
\Psi(\cdot)=\frac{1}{2 \lambda^{2}}+C \sum_{i=1}^{n} V\left(y_{i}, \frac{f\left(\mathbf{x}_{i}\right)}{\lambda}\right)+\mu\|\mathbf{z}\|_{1} .
$$

The first term in (12) represents the (inverse) functional margin. In comparison, the first term of (11) represents the (inverse) geometric margin. Recall, in SVM the norm is minimized as the functional margin is held constant at 1 to fix the scale of support vector coefficients. However, in the feature selection subproblem, support vector coefficients are already fixed. Thus we simply maximize the distance from the decision surface, corresponding to the functional margin. This allows greater flexibility in our search for optimal features and can further avoid suboptimal minima.

The procedure is summarized in Algorithm 3. We use the bound constrained trustregion algorithm to solve the explicit feature selection (EFS) subproblem. We set $\mathbf{z}=\mathbf{z}^{k}$ and $\lambda=1 / \sqrt{\sum_{i, j=1}^{n} y_{i} y_{j} \bar{u}_{i}^{k} \bar{u}_{j}^{k} K\left(Z^{k} \mathbf{x}_{i}, Z^{k} \mathbf{x}_{j}\right)}$ as initial points in step 6. Since we are 
mainly interested in feature selection, we use a weaker stopping criteria based on the zero norm of the weight vector. ${ }^{4}$ Compared to the full-space approach (Section 3 ), the explicit margin $\mathrm{AO}$ approach is more efficient. In addition, by focussing on improving the margin directly - a critical quantity for generalization - it further improves solution quality.

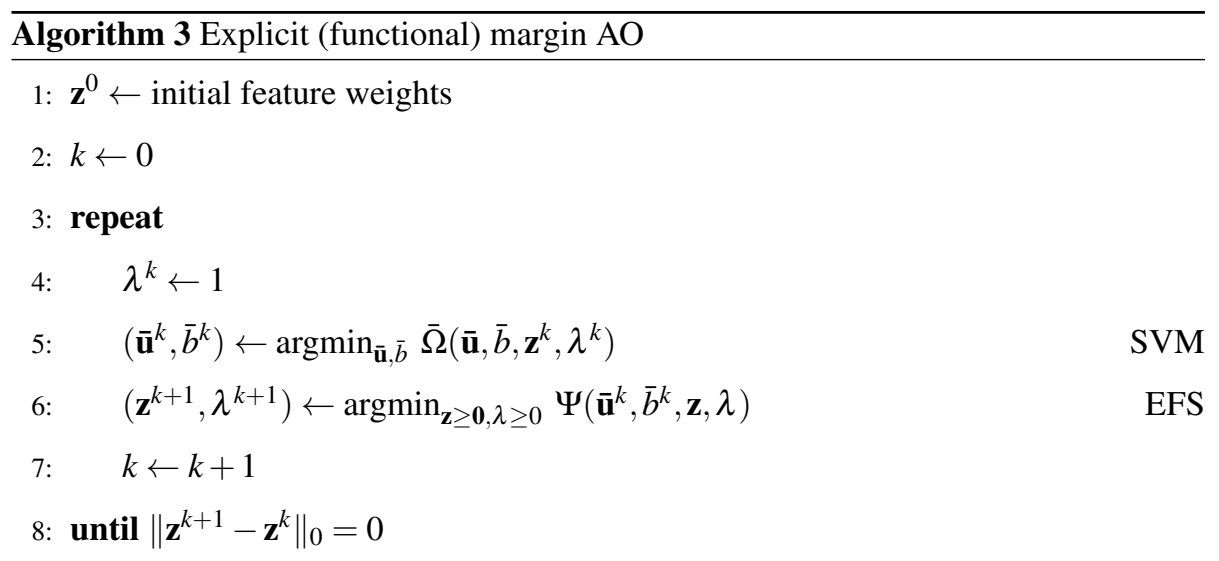

\section{Experiments}

In this section we evaluate our Full-Space (FULL-FS, Section 3) and AO-Explicit feature selection (AO-EFS, Section 4.3) methods on various datasets. We compare results with the state-of-the-art embedded feature selection algorithm, GMKL (Varma and Babu, 2009). On a simulated dataset (Thompson, 2006) we show that GMKL can fail to find the correct subset of features, while FULL-FS recovers a better solution and AO-EFS recovers the correct solution. On several other real datasets we show that our methods perform better than GMKL by $8-14 \%$ on average in terms of test error and with a reduction of $16-28 \%$ of features. We also demonstrate that FULL-FS and AO-EFS improve upon other leading filter and wrapper approaches in ranking relevant features.

\footnotetext{
${ }^{4}$ In our computation a component is considered zero if its absolute value is less than $0.01 \times \max _{k}\left|z_{k}\right|$.
} 


\subsection{Comparison to GMKL}

We optimize features using the same radial-basis kernel when comparing with GMKL. ${ }^{5}$

$$
K\left(Z \mathbf{x}_{i}, Z \mathbf{x}_{j}\right)=\exp \left(-\sum_{k=1}^{d}\left(z_{k} x_{i k}-z_{k} x_{j k}\right)^{2}\right),
$$

We use a 1-norm penalty on feature weights, $\mu\|\mathbf{z}\|_{1}$, similar to FULL-FS and AOEFS. All datasets are standardized to zero mean and unit variance and we always start with an initial feature weight vector of ones. The two parameters, $C$ and $\mu$, are determined by cross-validation over $\left(\log _{2} C, \log _{2} \mu\right)$ space at grid points $[-5,-4, \ldots, 14,15]$ $\times[-10,-8, \ldots, 8,10]$. We also compare results with regular SVM using the entire set of features. For SVM we use a radial basis kernel with width $\sigma$,

$$
K\left(\mathbf{x}_{i}, \mathbf{x}_{j}\right)=\exp \left(-\frac{\sum_{k=1}^{d}\left(x_{i k}-x_{j k}\right)^{2}}{\sigma^{2}}\right)
$$

and cross-validate over $\left(\log _{2} C, \log _{2} \sigma\right)$ at $[-5,-4, \ldots, 14,15] \times[-10,-8, \ldots, 8,10]$.

\subsubsection{Normally Distributed Clusters on Cubes}

In this example we evaluate feature selection using a simulated dataset. Normally distributed clusters on cubes (NDCC) generates nonlinearly separable data by sampling from multivariate normal distributions with centers at the vertices of three concentric 1-norm cubes (Thompson, 2006). An example with 2-dimensional cubes is shown in Figure 2. The distribution at each vertex uses a different (randomly generated) covariance matrix. Some centers generate a relatively small number of points, while others generate a relatively large number of points. Points around opposing vertices of each cube are assigned to opposite classes preventing linear separation.

In our experiment we generate data at vertices of 20-dimensional cubes and add 100 noisy features by sampling from a normal distribution. Thus the data contains a total of 120 features of which 20 are informative. This is a challenging dataset for feature selection because of the high degree of nonlinear interaction among informative features. Methods which rely on marginal contributions of features will perform poorly since projection to any single dimension will not reveal class separation.

\footnotetext{
${ }^{5}$ Implementation available at http://research.microsoft.com/en-us/um/people/manik/code/gmkl/download.html
} 


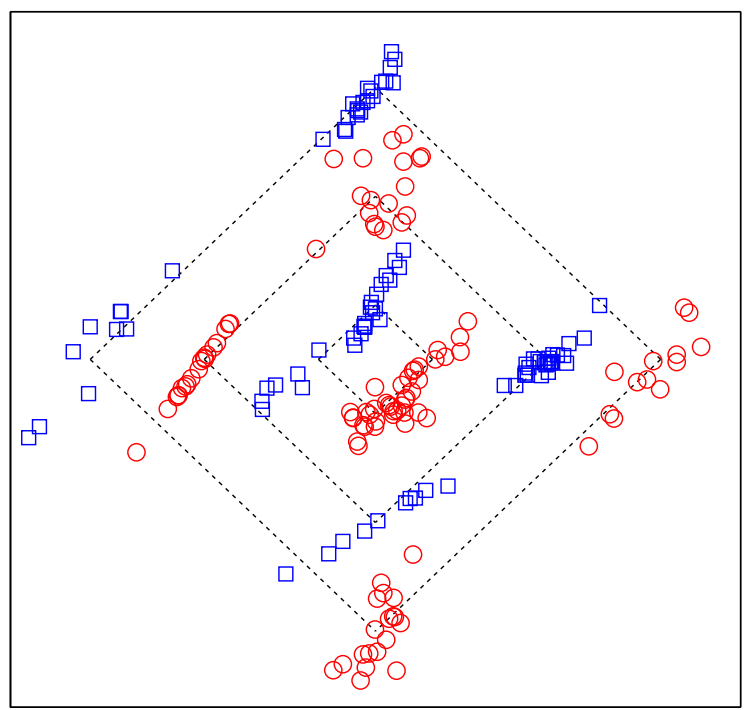

Figure 2: NDCC dataset example in 2-dimensions shown with the underlying 1-norm cubes.

We generate 200 training points, 200 validation points and 1000 testing points. Table 1 shows test error results using SVM, GMKL, FULL-FS and AO-EFS, along with the number of correct and incorrect features identified by each method. Note standard SVM is unable to detect a useful model since noisy features drown out any signal. This is clearly an example where feature selection is necessary in order to recover a meaningful model. The best parameter choice for GMKL, determined by cross-validation, yields 15 correct and 6 incorrect features resulting in a test error of $32.0 \%$. In comparison, FULL-FS is able to recover 17 correct features with 1 incorrect one and obtains $16.5 \%$ test error. Finally, AO-EFS is able to identify all 20 features with no incorrect ones and obtains the lowest test error of $10.5 \%$. We also observe that AO-EFS achieves a lower objective value and does not get stuck at suboptimal solutions.

\subsubsection{Gender Identification}

In this example, we try to identify gender from face images in the FEI database (Thomaz and Giraldi, 2010). The database consists of 200 different individuals collected from 


\begin{tabular}{lrrrr}
\hline & & & \multicolumn{2}{c}{ Number of Features } \\
\cline { 4 - 5 } & Objective & Test Error & Correct & Incorrect \\
\hline SVM & 106.1 & $44.3 \%$ & 20 & 100 \\
GMKL & 86.1 & $32.0 \%$ & 15 & 6 \\
FULL-FS & 75.2 & $16.5 \%$ & 17 & 1 \\
AO-EFS & 62.4 & $10.5 \%$ & 20 & 0 \\
\hline
\end{tabular}

Table 1: 20-dimensional NDCC dataset feature selection results. The objective value, test error and the number of correct and incorrect features are shown.

students and staff at FEI between the ages of 19 and 40. There are 100 male and 100 female subjects. Each image in the database has been aligned to a common template so that pixel-wise features correspond roughly to the same location across all subjects. Images are normalized, equalized, cropped and have been scaled down to have dimensions $18 \times 15$. Thus each image consists of 270 pixels of grey scale intensity. Figure 3 shows a few examples from the dataset.
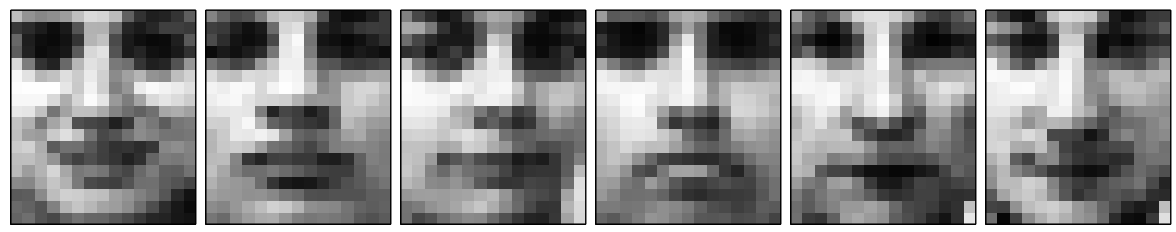

Figure 3: Example of a few processed images in the FEI faces dataset.

We follow standard experimental setup and use 167 images for training and 33 for out-of-sample testing. Results are averaged over 5 random splits of the data to reduce variance. Parameters are tuned by running 10-fold cross-validation on the training set for each split.

Table 2 shows the feature selection results. AO-EFS achieves an error of $11.0 \%$ using on average 16 features. In comparison, FULL-FS achieves an error of $11.9 \%$ using 19 features and GMKL performs comparatively worse with an error of $12.6 \%$ 
using 31 features. Regular SVM obtains an error of 10.8\%. SVM results are obtained using all 270 features. AO-EFS can obtain similar generalization error with approximately 17 times compression factor. Figure 4 shows the average male and female faces superimposed with the features identified by GMKL, FULL-FS and AO-EFS.

\begin{tabular}{lrr}
\hline & Test Error(\%) & Av. \# of Features \\
\hline SVM & $10.8 \pm 0.8$ & 270.0 \\
GMKL & $12.6 \pm 1.4$ & 31.4 \\
FULL-FS & $11.9 \pm 0.9$ & 19.1 \\
AO-EFS & $11.0 \pm 0.6$ & 16.2 \\
\hline
\end{tabular}

Table 2: Test error and average number of features obtained on the FEI faces dataset.

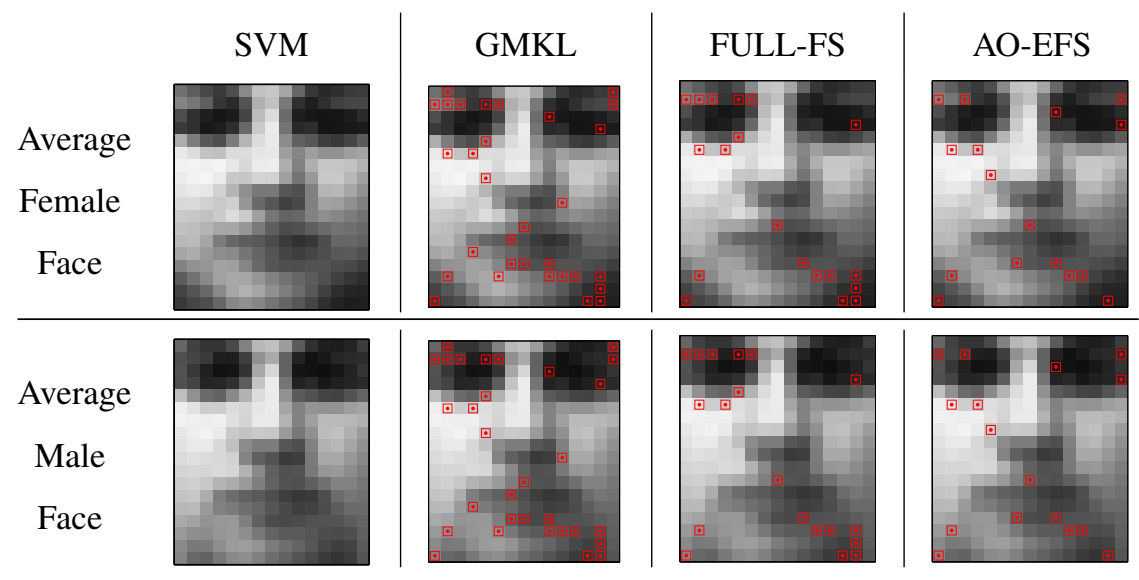

Figure 4: The average male and female faces in the FEI dataset superimposed with the features identified by GMKL, FULL-FS and AO-EFS. Note that SVM uses all 270 features.

\subsubsection{Other datasets}

We compare results on several other datasets obtained from UCI repository (Frank and Asuncion, 2010). Two-thirds of the observations are used for training and the remaining one-third for out-of-sample testing. Results are averaged over 5 (stratified) 
random splits of the data. Parameters are tuned by running 10 -fold cross-validation on the training set for each split. This methodology is used for all datasets, except Madelon. Madelon was used in the NIPS 2003 Feature Selection Challenge ${ }^{6}$ and comes with separate training, validation and testing sets.

Table 3 summarizes the feature selection results. Average test errors and corresponding average number of features are shown for each dataset. FULL-FS and AOEFS improve test error on average by $8 \%$ and $14 \%$ compared to GMKL, while using $16 \%$ and $28 \%$ fewer features, respectively. AO-EFS performs slightly better than FULL-FS in terms of test error and number of features used. A regular SVM using uniform feature weights generally yields similar performance, though FULL-FS and AO-EFS use significantly fewer features. The exception is the Madelon dataset. Madelon is constructed specifically to evaluate multivariate feature selection and by design contains many noisy features, which lead to poor SVM performance.

\subsection{Feature Ranking Comparison}

In this section we evaluate the ability of FULL-FS and AO-EFS to rank features. We compare with GMKL as well as three other popular feature selection methods, described below: ${ }^{7}$

- Mutual Information (MI): A filter method, which uses the mutual information score between candidate features and the output class as a basis to rank features (Zaffalon and Hutter, 2002). For discrete random variables, mutual information is given by

$$
I(\pi)=\sum_{i} \sum_{j} \pi_{i j} \log \frac{\pi_{i j}}{\pi_{i} \pi_{j}}
$$

where $\pi_{i j}$ is the probability (frequency) of jointly observing events $i$ and $j$, and $\pi_{i}=\sum_{j} \pi_{i j}$ and $\pi_{j}=\sum_{i} \pi_{i j}$ are the marginal probability of events. Continuous features are binned to a discrete set corresponding to index $i$, while $j$ indexes the

\footnotetext{
${ }^{6}$ http://www.nipsfsc.ecs.soton.ac.uk/

${ }^{7} \mathrm{We}$ use the implementations provided in the Spider machine learning toolbox for these algorithms, http://people.kyb.tuebingen.mpg.de/spider/.
} 


\begin{tabular}{|c|c|c|c|c|c|c|c|c|c|}
\hline & \multirow[b]{2}{*}{$n$} & \multicolumn{4}{|c|}{ Test Error } & \multicolumn{4}{|c|}{ Average Number of Features } \\
\hline & & SVM & GMKL & FULL-FS & AO-EFS & SVM & GMKL & FULL-FS & AO-EFS \\
\hline Sonar & 208 & $21.1 \pm 1.0$ & $16.8 \pm 1.1$ & $16.0 \pm 1.1$ & $14.9 \pm 0.8$ & 60.0 & 12.9 & 13.9 & 12.3 \\
\hline Ion & 351 & $5.1 \pm 0.2$ & $6.0 \pm 0.4$ & $5.3 \pm 0.8$ & $5.3 \pm 0.4$ & 33.0 & 10.6 & 10.7 & 7.4 \\
\hline S.A. Heart & 462 & $27.8 \pm 0.9$ & $30.6 \pm 1.1$ & $29.9 \pm 0.7$ & $28.7 \pm 0.8$ & 9.0 & 5.4 & 3.9 & 3.8 \\
\hline Musk & 476 & $7.6 \pm 0.7$ & $9.3 \pm 0.8$ & $9.7 \pm 0.7$ & $7.0 \pm 0.6$ & 166.0 & 29.9 & 32.4 & 27.5 \\
\hline Wdbc & 569 & $3.7 \pm 0.5$ & $5.3 \pm 0.4$ & $3.8 \pm 0.4$ & $3.8 \pm 0.3$ & 30.0 & 6.3 & 6.6 & 4.5 \\
\hline Aust. Credit & 690 & $13.5 \pm 0.7$ & $14.3 \pm 0.5$ & $13.4 \pm 0.4$ & $13.0 \pm 0.3$ & 14.0 & 9.6 & 5.7 & 7.0 \\
\hline German Credit & 1000 & $23.2 \pm 0.5$ & $23.6 \pm 0.8$ & $23.1 \pm 0.4$ & $23.4 \pm 0.4$ & 24.0 & 12.0 & 10.4 & 9.8 \\
\hline Madelon & 2000 & 40.7 & 7.7 & 7.2 & 7.0 & 500.0 & 14.0 & 10.0 & 8.0 \\
\hline \multicolumn{4}{|c|}{ Avg. improvement rel. to GMKL } & $7.7 \%$ & $13.6 \%$ & & & $15.8 \%$ & $27.8 \%$ \\
\hline
\end{tabular}

Table 3: Feature selection results on UCI datasets comparing test error and average number of features used. Note, SVM uses all the features in the dataset. $n$ is the number of examples in the dataset. Refer to text for experiment methodology. The last row shows the average percentage improvement compared to GMKL. 
binary class output. Higher values of $I(\pi)$ imply greater dependence between the feature and output.

- Relief: A multivariate filter method, which estimates feature relevance by determining how well they distinguish classes between nearby points (Kira and Rendell, 1992). At each iteration a point is chosen and the weight for each feature is updated according to the distance of the point to its nearest neighbor from the same class (hit) and nearest neighbor from the other class (miss). The final score of a feature is the ratio between the average distance (in projection on that feature) to the nearest miss and nearest hit over all examples.

- Recursive Feature Elimination (RFE): A wrapper method that uses a greedy approach to eliminate features, one at a time, that decrease the margin the least (Guyon et al., 2002). An SVM is trained at each iteration, and the (inverse) margin is computed: $W^{2}(\mathbf{u})=\sum u_{i} u_{j} y_{i} y_{j} K\left(\mathbf{x}_{i}, \mathbf{x}_{j}\right)$. For each feature $l, W_{(-l)}^{2}(\mathbf{u})=$ $\sum u_{i} u_{j} y_{i} y_{j} K\left(\mathbf{x}_{i}^{-l}, \mathbf{x}_{j}^{-l}\right)$ is computed, where $\mathbf{x}_{i}^{-l}$ means training point $i$ with feature $l$ removed. The feature with the smallest value of $\left|W^{2}(\mathbf{u})-W_{(-l)}^{2}(\mathbf{u})\right|$ is removed. Repeated application of the procedure results in a ranking of features.

For embedded feature selection methods (i.e. GMKL, FULL-FS, AO-EFS), instead of varying parameter $\mu$ to select the required number of features, we obtain rankings by taking the top ranked components of $\mathbf{z}$ at a fixed $C$ and $\mu$. $C$ and $\mu$ are chosen by cross-validation to minimize classification error. Similar methodology was used by Varma and Babu (2009).

We show test error results versus the number of selected features in Figures 5 to 14. Each figure corresponds to a dataset used in Section 5.1. For a given number of features, we select the top ranked features and relearn an SVM classifier using only the selected features. We use a radial basis kernel and cross-validate to determine optimal SVM parameters, $C$ and $\sigma$, for the reduced feature set. Apart from the NDCC and Madelon datasets, each test error point is obtained by averaging results over five trials. In each trial, two-thirds of the data is used for training and one-third for testing. Parameters $C$ and $\sigma$ are tuned by 10 -fold cross-validation on the training set. NDCC and Madelon use separate training, validation and testing sets. 


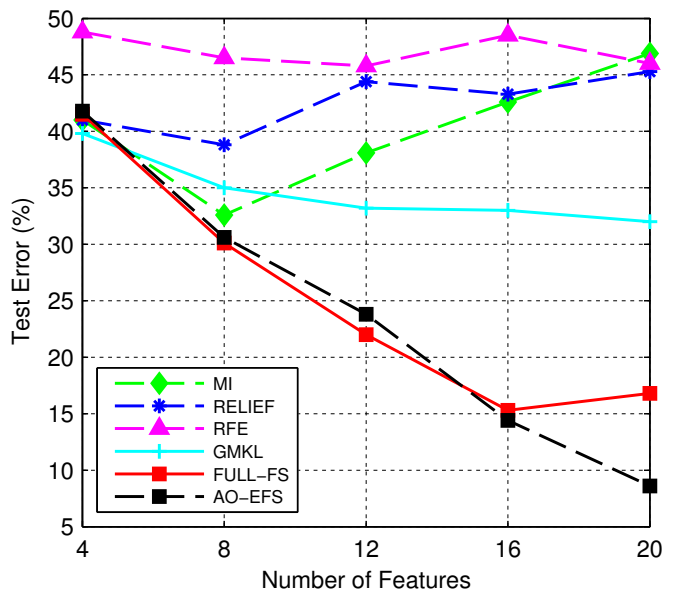

Figure 5: Test error as a function of number of features selected for NDCC.

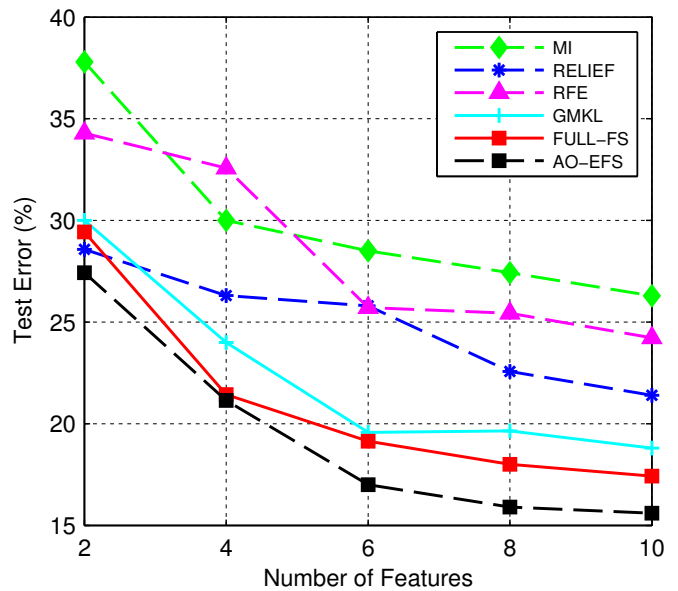

Figure 7: Test error as a function of number of features selected for Sonar.

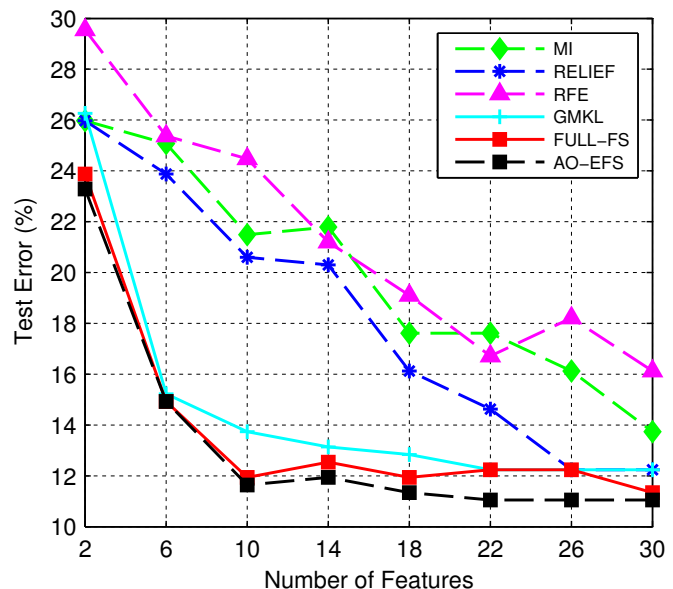

Figure 6: Test error as a function of number of features selected for FEI Faces.

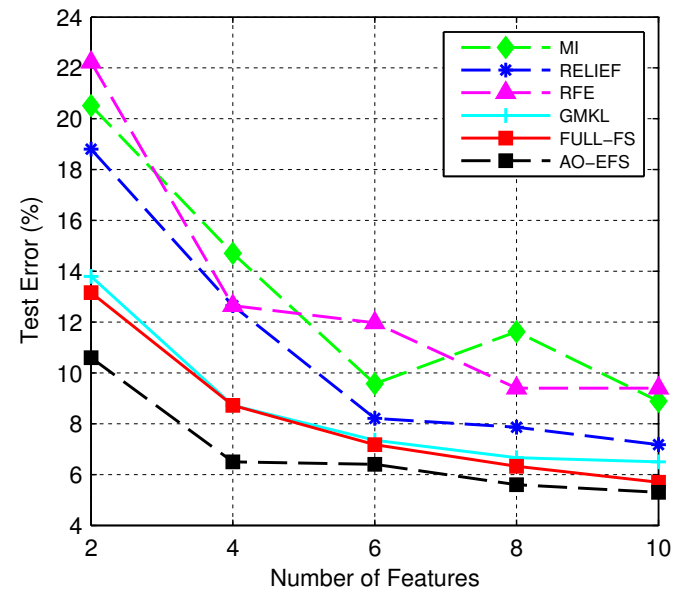

Figure 8: Test error as a function of number of features selected for Ion. 


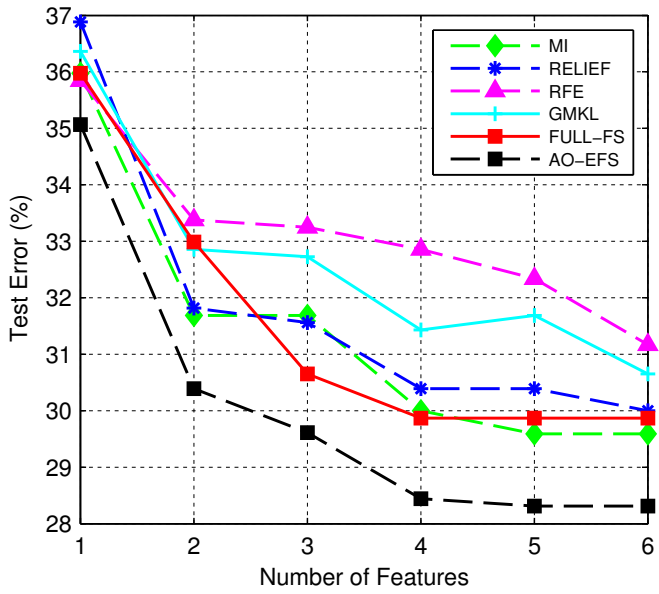

Figure 9: Test error as a function of number of features selected for S.A. Heart.

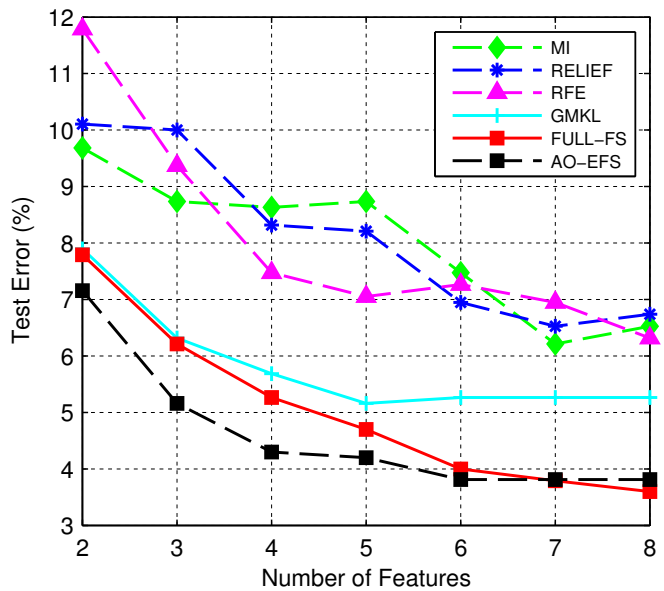

Figure 11: Test error as a function of number of features selected for Wdbc.

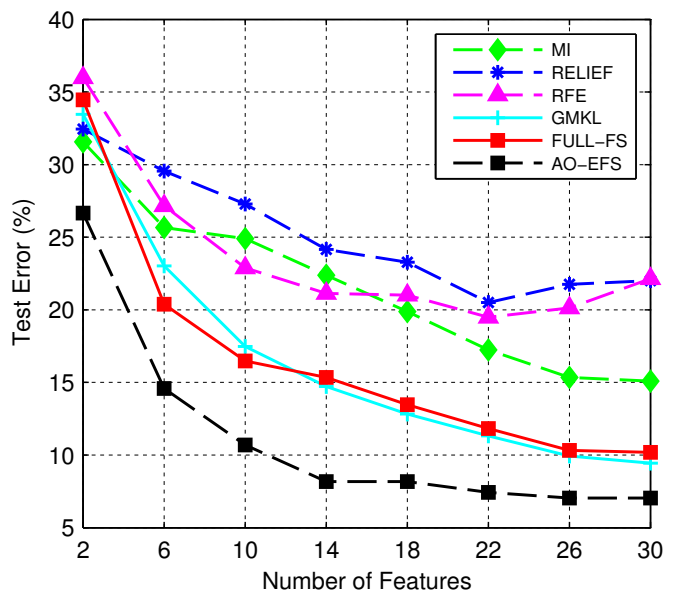

Figure 10: Test error as a function of number of features selected for Musk.

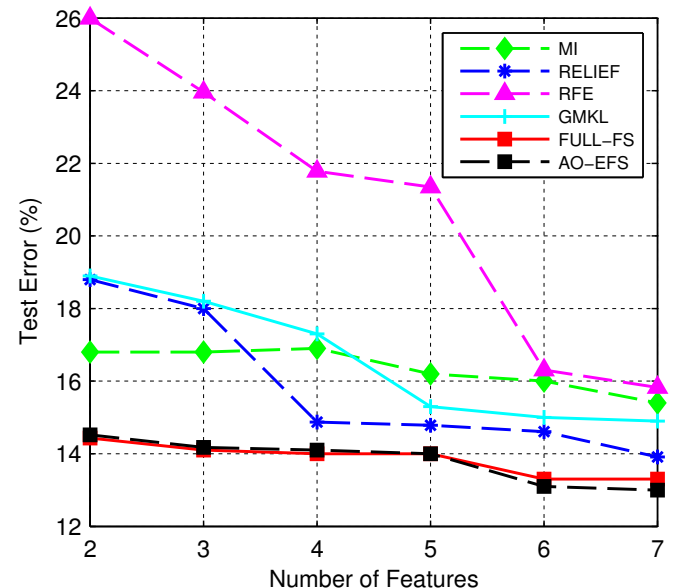

Figure 12: Test error as a function of number of features selected for Aust. Credit. 


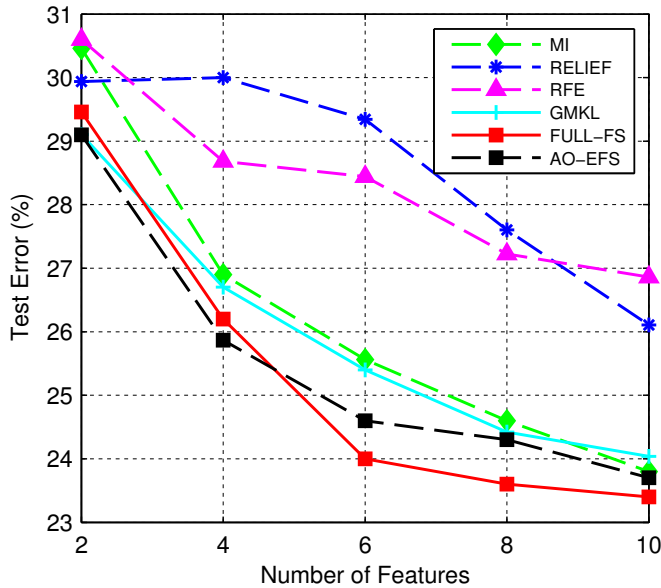

Figure 13: Test error as a function of number of features selected for German Credit.

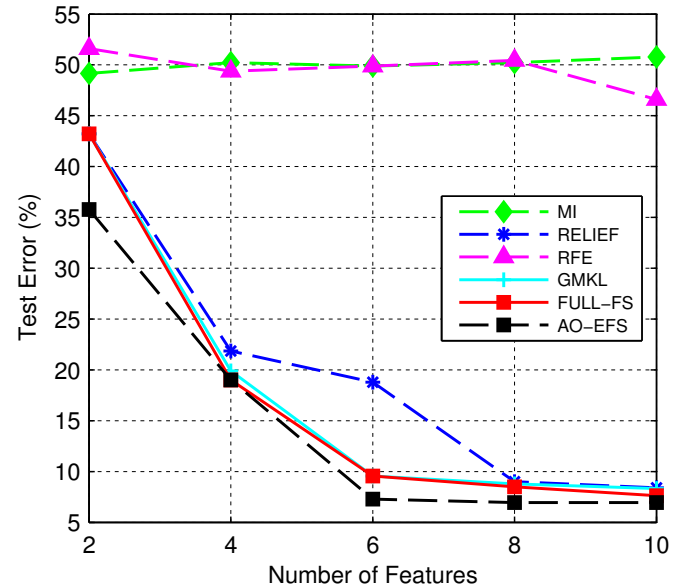

Figure 14: Test error as a function of number of features selected for Madelon.

The results show that embedded methods generally perform better than filter and wrapper methods, as expected. This is more prominent in the NDCC and Madelon datasets, where there is a complex multivariate relationship among informative features. RELIEF performs well on Madelon, since it is able to capture multivariate relationships, but is not as effective on other datasets. MI performs well when single features are independently significant, for example in German Credit data, but is unable to identify multivariate relationships. Among the embedded methods, AO-EFS performs the best, followed closely by FULL-FS, and then GMKL. In particular, we see GMKL is not as effective on some datasets, namely NDCC, S.A. Heart, Wdbc, and Aust. Credit, compared to FULL-FS and AO-EFS.

\section{Conclusion}

We developed an effective algorithm to solve the non-convex optimization problem that results from embedding feature selection in nonlinear SVMs. We solve the primal embedded SVM problem using a trust region method for a bound constrained problem, which is more suitable for non-convex optimization than line-search methods. The trust region algorithm uses local second order approximate models and can guarantee 
convergence to a minimizer. For computational efficiency, we apply an alternating optimization (AO) framework. We show a naive application of AO can lead to iterates being trapped at saddle points. We extend the space in which $\mathrm{AO}$ is performed with an auxiliary variable corresponding to the margin. Sharing the margin variable between AO subproblems reduces saddle point convergence. We further improve solution quality by directly maximizing the functional margin, instead of the geometric margin, in the feature selection subproblem. This focusses on maximizing margin, while permitting greater flexibility, as we optimize over the feature space.

We compare our proposed methods to GMKL, the state-of-the-art embedded SVM feature selection method. GMKL uses a gradient descent algorithm, which does not guarantee convergence to a minimizer for a non-convex problem and can be susceptible to suboptimal solutions. On a simulated dataset we show that GMKL can get stuck at poor solutions and is unable to recover the correct feature subset. On several other real datasets we show that our methods improve upon GMKL by $8-14 \%$ in test error while further reducing features by $16-28 \%$. We also show how our methods outperform other leading filter and wrapper approaches in ranking features.

While our algorithm has been described in the context of feature selection, it can be generalized to non-convex multiple kernel learning. For future work, we hope to further investigate theoretical and convergence properties of sharing a suitably chosen auxiliary variable under a block-coordinate $\mathrm{AO}$ scheme.

\section{References}

Bezdek, J. C., Hathaway, R. J., 2002. Some notes on alternating optimization. In: Proceedings of the 2002 AFSS International Conference on Fuzzy Systems (AFSS '02). pp. 288-300.

Boser, B. E., Guyon, I. M., Vapnik, V. N., 1992. A training algorithm for optimal margin classifiers. In: Proceedings of the fifth annual workshop on Computational learning theory. COLT '92. ACM, pp. 144-152.

Boyd, S., Vandenberghe, L., 2004. Convex Optimization. Cambridge University Press. 
Bradley, P. S., Mangasarian, O. L., 1998. Feature selection via concave minimization and support vector machines. In: Machine Learning Proceedings of the Fifteenth International Conference(ICML 98). pp. 82-90.

Byun, H., Lee, S.-W., 2002. Applications of support vector machines for pattern recognition: A survey. In: Pattern Recognition with Support Vector Machines. pp. 213236.

Chan, A. B., Vasconcelos, N., Lanckriet, G. R. G., 2007. Direct convex relaxations of sparse svm. In: Proceedings of the 24th international conference on Machine learning. ICML '07. ACM, pp. 145-153.

Chapelle, O., Vapnik, V., Bousquet, O., Mukherjee, S., 2002. Choosing multiple parameters for support vector machines. Mach. Learn. 46 (1-3), 131-159.

Coleman, T. F., Li, Y., 1996. An interior trust region approach for nonlinear minimization subject to bounds. SIAM Journal on Optimization 6 (2), 415-425.

Cortes, C., Vapnik, V., 1995. Support-vector networks. Machine Learning 20 (3), 273297.

Courant, R., Hilbert, D., 1953. Methods of Mathematical Physics. Interscience.

Danskin, J., 1967. The Theory of Max-Min and its Application to Weapons Allocation Problems.

Fan, R.-E., Chen, P.-H., Lin, C.-J., 2005. Working set selection using second order information for training support vector machines. Journal of Machine Learning Research 6, 1889-1918.

Frank, A., Asuncion, A., 2010. UCI machine learning repository.

URL http://archive.ics.uci.edu/ml

Fung, G. M., Mangasarian, O. L., 2004. A feature selection newton method for support vector machine classification. Computational Optimization and Applications 28 (2), $185-202$. 
Grippo, L., Sciandrone, M., 2000. On the convergence of the block nonlinear GaussSeidel method under convex constraints. Operations Research Letters 26, 127-136.

Guyon, I., Elisseeff, A., 2003. An introduction to variable and feature selection. Journal of Machine Learning Research 3, 1157-1182.

Guyon, I., Weston, J., Barnhill, S., Vapnik, V., 2002. Gene selection for cancer classification using support vector machines. Machine Learning 46 (1-3), 389-422.

Kira, K., Rendell, L., 1992. A practical approach to feature selection. In: ML92: Proceedings of the ninth international workshop on Machine learning. pp. 249-256.

Marchiori, E., 2005. Feature selection for classification with proteomic data of mixed quality. In: In Proceedings of the 2005 IEEE Symposium on Computational Intelligence in Bioinformatics and Computational Biology. pp. 385-391.

Mercer, J., 1909. Functions of positive and negative type and their connection with the theory of integral equations. Philos. Trans. Royal Soc. (A) 83 (559), 69-70.

Nguyen, X., Wainwright, M. J., Jordan, M. I., 2009. On surrogate loss functions and f-divergences. Annals of Statistics 37 (2), 876-904.

Platt, J. C., 1999. Advances in kernel methods. MIT Press, Ch. Fast training of support vector machines using sequential minimal optimization, pp. 185-208.

Rosset, S., Zhu, J., Hastie, T., 2003. Margin maximizing loss functions. In: Advances in Neural Information Processing Systems (NIPS 15). MIT Press.

Scholkopf, B., Smola, A. J., 2002. Learning with Kernels. MIT Press.

Schölkopf, B., Tsuda, K., Vert, J. P. (Eds.), 2004. Kernel Methods in Computational Biology. MIT Press.

Tan, M., Wang, L., Tsang, I. W., 2010. Learning sparse svm for feature selection on very high dimensional datasets. In: Proceedings of the 27 th International Conference on Machine Learning (ICML-10). pp. 1047-1054. 
Thomaz, C. E., Giraldi, G. A., 2010. A new ranking method for principal components analysis and its application to face image analysis. Image and Vision Computing $28(6), 902-913$.

Thompson, M. E., 2006. NDCC: normally distributed clustered datasets on cubes. Www.cs.wisc.edu/dmi/svm/ndcc/.

Tibshirani, R., 1996. Regression shrinkage and selection via the lasso. J. Roy. Statist. Soc. Ser. B 58 (1), 267-288.

Vapnik, V. N., 1998. Statistical Learning Theory, 1st Edition. Wiley.

Varma, M., Babu, B. R., 2009. More generality in efficient multiple kernel learning. In: Proceedings of the 26th International Conference on Machine Learning (ICML '09). pp. 1065-1072.

Šikonja, M. R., Kononenko, I., 2003. Theoretical and empirical analysis of ReliefF and RReliefF. Machine Learning 53 (1-2), 23-69.

Weston, J., Elisseeff, A., Schölkopf, B., Tipping, M., 2003. Use of the zero norm with linear models and kernel methods. Journal of Machine Learning Research 3, 14391461.

Zaffalon, M., Hutter, M., 2002. Robust feature selection by mutual information distributions. In: Proceedings of the Eighteenth conference on Uncertainty in artificial intelligence. UAI'02. pp. 577-584.

Zhu, J., Rosset, S., Hastie, T., Tibshirani, R., 2003. 1-norm support vector machines. In: Neural Information Processing Systems. Vol. 16. 